Research Paper

\title{
Niclosamide sensitizes nasopharyngeal carcinoma to radiation by downregulating Ku70/80 expression
}

\author{
Jinging Li $i^{*}$, Haiwen Li*, Dechao Zhan*, Mei Xiang, Jun Yang, Yufang Zuo, Yin Yu, Hechao Zhou, Danxian \\ Jiang, Haiqing Luo, Zihong Chen, Zhonghua $\mathrm{Yu}^{\bowtie}$, Zumin $\mathrm{Xu}^{凶}$ \\ Cancer Center, Affiliated Hospital of Guangdong Medical University, Zhanjiang 524000, Guangdong Province, China. \\ * These authors have contributed equally to this study. \\ $\triangle$ Corresponding authors: Zhonghua Yu, MD, Cancer Center, Affiliated Hospital of Guangdong Medical University; 57 South Renmin Road, Zhanjiang 524000, \\ Guangdong Province, China; Phone: 07592387813; Fax: 07592387813; E-mail: zhonghua_yu@126.com; Zumin Xu, MD\&PhD, Cancer Center, Affiliated Hospital \\ of Guangdong Medical University; 57 South Renmin Road, Zhanjiang 524000, Guangdong Province, China; Phone: 075923874448; Fax: 07592387448; E-mail: \\ zuminxu@gdmu.edu.cn. \\ (c) Ivyspring International Publisher. This is an open access article distributed under the terms of the Creative Commons Attribution (CC BY-NC) license \\ (https://creativecommons.org/licenses/by-nc/4.0/). See http://ivyspring.com/terms for full terms and conditions.
}

Received: 2017.05.10; Accepted: 2017.12.13; Published: 2018.02.06

\begin{abstract}
The aim of the present study was to investigate whether niclosamide could sensitize the nasopharyngeal carcinoma cells to radiation and further explore the underlying mechanisms. CCK-8 assay was used to determine the effect of niclosamide on the proliferation of NPC cells. Colony formation assay was used to evaluate the radiosensitizing effect of niclosamide on NPC cells. Flow cytometry analysis was used to determine the apoptosis of NPC cells induced by niclosamide. Immunofluorescent staining was used to detect the formation of $\mathrm{Y}-\mathrm{H} 2 \mathrm{AX}$ foci and the localization of Ku70/80 proteins in NPC cells. Real-time PCR quantification analysis was used to examine the level of Ku70/80 mRNA. DNA damage repair-related proteins were detected by western blot analysis. Our results showed that niclosamide markedly suppressed the proliferation of NPC cells. Niclosamide pretreatment followed by irradiation reduced the colony forming ability of NPC cells. Niclosamide in combination with irradiation significantly increased the apoptotic rate of NPC cells. Niclosamide significantly reduced the transcriptional level of K70/80 but not the translocation of $\mathrm{Ku} 70 / 80$ protein induced by irradiation. In conclusion, our study demonstrated that niclosamide could inhibit the growth of NPC cells and sensitize the NPC cells to radiation via suppressing the transcription of Ku70/80.
\end{abstract}

Key words: niclosamide; nasopharyngeal carcinoma; radiosensitization; DNA damage repair; Ku70/80

\section{Introduction}

Nasopharyngeal carcinoma (NPC) is one of the most common head and neck malignancies in Southeast Asia, especially in Southern China ${ }^{l}$. Radiotherapy is the mainstay treatment modality for NPC. With the development of intensity-modulated radiation therapy technique, more than $85 \%$ locoregional control has been consistently reported ${ }^{2}$. However, approximately $20 \%$ of NPC patients develop local recurrence after treatment, and resistance to radiotherapy is a significant component of cancer treatment failure ${ }^{3}$. Thus, it is urgent to find new drugs to improve the outcome of radiotherapy for NPC.
Niclosamide is a teniacide in the antihelmintic family that is especially effective against cestodes, which infects humans. Niclosamide has been FDA approved for such indications and has been used in humans for nearly 50 years $^{4,5}$. Recently, anti-cancer activity of niclosamide has been demonstrated in types of cancers, including breast cancer, prostate cancer, colon cancer, ovarian cancer, multiple myeloma, acute myelogenous leukemia, glioblastoma, head and neck cancer and lung cancer cells ${ }^{6-10}$. Niclosamide is able to block multiple signaling pathways that govern cancer initiation and progression $^{11-13}$. However, the impact of niclosamide 
on the response of NPC to irradiation remains unknown.

In the present study, we investigated whether niclosamide could sensitize the nasopharyngeal carcinoma cells to radiation and further explored the underlying mechanisms.

\section{Materials and Methods}

\section{Cell cultures}

Two undifferentiated human NPC cell lines, CNE-2 and HONE-1, were maintained by our laboratory and cultured in RPMI-1640 medium (HyClone, Logan, UT, USA) supplemented with 10\% FBS (fetal bovine serum) (Biological Industries), 100 $\mathrm{U} / \mathrm{ml}$ penicillin and $100 \mathrm{U} / \mathrm{ml}$ streptomycin in a humid atmosphere of $5 \% \mathrm{CO} 2$ at $37^{\circ} \mathrm{C}$.

\section{Irradiation condition}

The NPC cells were exposed to $8 \mathrm{MV}$ of X-rays with various doses of irradiation (0-8Gy) using a linear accelerator (Eleketa, Stockholm, Sweden) with the source-skin-distance technique $(\mathrm{SSD}=100 \mathrm{~cm})$. The depth was set at $1.5 \mathrm{~cm}$ to the bottom of the 6-well plate or 6-cm dishes.

\section{Cell proliferation assay}

Cells in the early log phase were trypsinized and plated in a 96-well plate at a density of $1 \times 10^{4}$ cells per well. Twenty-four hours later, the medium was removed and replaced with fresh medium with niclosamide (Sigma-Aldrich, St. Louis, MO, USA) at the indicated concentrations $(0.25,0.5,1,2,4,8,16,32$ $\mathrm{mM}$ ) for 24 or $48 \mathrm{~h}$ in the presence of $1 \%$ FBS. Cell density was measured using the CCK-8 (Dojindo Molecular Technologies, Kumamoto, Japan) assay following the manufacturer's instructions. The absorbance of each well was determined at $450 \mathrm{~nm}$ using a microplate reader. The percentage of surviving cells from each group relative to the control was defined as the proliferation rate. For these studies, all experiments were repeated at least three times.

\section{Colony formation assay}

Cells in early log phase were trypsinized and plated in 6-well plates at 200, 400, 1000, 2000 and 4000 cells per well and cultured overnight to allow for cell attachment. Then cells were treated with or without niclosamide for $24 \mathrm{~h}$ prior to administration of irradiation with the exposure dose corresponding to 0 , 2, 4, 6, and $8 \mathrm{~Gy}$. The cells were incubated for 10 days to allow for the formation of colonies. Cells were fixed and stained with $0.5 \%$ crystal violet (Sigma-Aldrich, St. louis, MO, USA), and colonies containing $>50$ cells were counted. Survival curves were fitted using the multi-target click model in Graph Pad Prism 5.0
(GraphPad Software Inc., La Jolla, CA, USA). Each point on the survival curve represents the mean surviving fraction from at least three independent experiments.

\section{Cell apoptosis assay}

For flow cytometry analysis, $1 \times 10^{5}$ cells were harvested after different treatment, collected by centrifugation (10 min, $2000 \mathrm{rcf}$ ) and washed three times with phosphate-buffered saline (PBS) at $4^{\circ} \mathrm{C}$. Prior to analysis, cells were resuspended in binding buffer (10 mM Hepes/ NaOH, pH 7.4, $140 \mathrm{mM} \mathrm{NaCl}$, $2.5 \mathrm{mM} \mathrm{CaCl}$ ). Cells were then incubated with $5 \mu \mathrm{l}$ Annexin V-FITC for $3 \mathrm{~min}$ and with $20 \mathrm{ng} / \mathrm{ml}$ propidium iodide in the dark for $15 \mathrm{~min}$. This suspension was analyzed by flow cytometry (Becton-Dickinson, San Jose, CA, USA). All data were collected and analyzed by BD FACSDiva software (version 6.1.3, BD Biosciences). The experiments were repeated three times independently and the results were presented as the mean \pm standard deviation.

\section{Immunofluorescent staining}

For immunofluorescent staining assay, cells (2.5 $\times 10^{5} /$ dish) were plated onto sterile coverslips, and were treated with or without niclosamide after 24 hours' incubation. Twelve hours later, radiotherapy and radiotherapy plus niclosamide cells were irradiated at a total dose of $6 \mathrm{~Gy}$. Cells were collected at the indicated time points $(1,4$ and $12 \mathrm{~h})$, washed, fi xed with methanol and then blocked with 5\% BSA before incubation in rabbit monoclonal anti- $\gamma-\mathrm{H} 2 \mathrm{AX}$ (Ser 139) (1:400; CST, Danvers, MA, USA) or rabbit monoclonal anti-Ku70 (1:100; abcam; CA; USA), and anti-Ku80 (1:400; CST, Danvers, MA, USA) antibody overnight at $4^{\circ} \mathrm{C}$. After rinsing with PBS three times, the coverslips were incubated in secondary anti-rabbit Alexa Fluor 488 antibody (1:500; Invitrogen, Camarillo, CA, USA) for $1 \mathrm{~h}$ at room temperature. Then cells were stained with DAPI (Sigma-Aldrich, St. Louis, MO, USA) for 15 min. Coverslips were then mounted onto slides with anti-fade mounting medium (Solarbio, Beijing, China). The images were visualized, and representative views of the cells were recorded by a confocal microscope (Leica TCS SP5, Wetzlar, Germany). For each treatment condition, the numbers of $\gamma-\mathrm{H} 2 \mathrm{AX}$ was counted for 50 cells at least. For the negative-control staining, the primary antibodies were omitted.

\section{Western blot analysis}

After treatment with niclosamide and/ or irradiation (6 Gy), samples for immunoblotting were collected as previously described ${ }^{14}$. The membrane was then incubated with the appropriate primary 
antibody, anti-ATR, anti-phospho-ATR (Ser428), anti-Ku70, anti-Ku80, (1:1,000; CST, Danvers, MA, USA), and $\beta$-actin (1:1,500; Beyotime Biotech, China). The protein of interest was detected with anti-rabbit or anti-mouse IgG-horseradish peroxidase-conjugated secondary antibody (1:1,500; Beyotime Biotech, China). The band intensities were measured using Image J 1.41 software (NIH, Bethesda, MD, USA). Data are presented as the relative protein levels normalized to $\beta$-actin, and the ratio of the control samples was taken as 1.0.

\section{Quantitative real-time PCR analysis}

To quantitate mRNA expression, total RNA was extracted from NPC cell lines pretreated with or without niclosamide with RNAiso Plus kits (Takara, Japan). The isolated total RNA was reverse transcribed using the PrimeScript ${ }^{\mathrm{TM}}$ RT reagent Kit with gDNA Eraser (Takara, Japan) according to the manufacturer's instructions. Relative expression was calculated via the comparative cycle threshold $(\mathrm{Ct})$ method using the expression of RNA as the reference. The sequence-specific forward primers for $\mathrm{Ku} 70 / \mathrm{Ku} 80$ and $\beta$-actin internal control were 5'-GCTCCTTGGTGGATGAGTTT-3' ' 5' -GGCTCTTTC CGCTATCTGC-3' and 5'-GGCGGCAACACCATGTA CCC-3' respectively. The reverse qPCR Primer were 5'-CTTGCTGATGTGGGTCTTCA-3', 5'-CGTCCATA CACAGCACAACA-3' and 5'-AGGGGCCGGACTCC GTCATACT. The amount of RNA was monitored with SYBR ${ }^{\circledR}$ Premix Ex Taq ${ }^{\mathrm{TM}}$ II (Perfect Real Time) (Takara, Japan). The reactions were performed on a LightCycler ${ }^{\circledR}$ (Roche Diagnostics, USA). The PCR conditions were $30 \mathrm{~s}$ at $95^{\circ} \mathrm{C}$, followed by 40 cycles at $95^{\circ} \mathrm{C}$ for $5 \mathrm{~s}$ and $60^{\circ} \mathrm{C}$ for $20 \mathrm{~s}$. Relative expression was calculated using the $2^{-\Delta \mathrm{CT}}$ method.

\section{Statistical analysis}

All data are expressed as mean values \pm SD. For two-group comparison, the Student's t-test method was used. For more than a two-group comparison, one-way ANOVA was used. SPSS 13.0 software was used for all statistical analyses (SPSS, Chicago, IL, USA). $P$ value less than 0.05 was considered statistically significant.

\section{Results}

\section{Niclosamide inhibited the proliferation of NPC cells in a dose- and time-dependent manner}

To test whether niclosamide could inhibit the proliferation of NPC cells, the CNE-2 and HONE-1 cells were treated with different concentrations of niclosamide as indicated (Fig. 1). The proliferation of these two cells was remarkably inhibited by niclosamide in dose- and time-dependent manner. Increasing concentrations of niclosamide and prolonged time from 24 to 48 hours resulted in significant reduction in the cell viability of these two cell lines. The $50 \%$ inhibitory concentrations $\left(\mathrm{IC}_{50}\right)$ of niclosamide in CNE-2 cells were $2.61 \pm 0.05 \mu \mathrm{mol} / \mathrm{L}$ at $24 \mathrm{~h}$ and $1.15 \pm 0.04 \mu \mathrm{mol} / \mathrm{L}$ at $48 \mathrm{~h}$, respectively; while the $\mathrm{IC}_{50}$ of niclosamide in HONE-1 cells were $1.87 \pm 0.28 \mu \mathrm{mol} / \mathrm{L}$ at $24 \mathrm{~h}$ and $0.74 \pm 0.02 \mu \mathrm{mol} / \mathrm{L}$ at $48 \mathrm{~h}$, respectively.
A

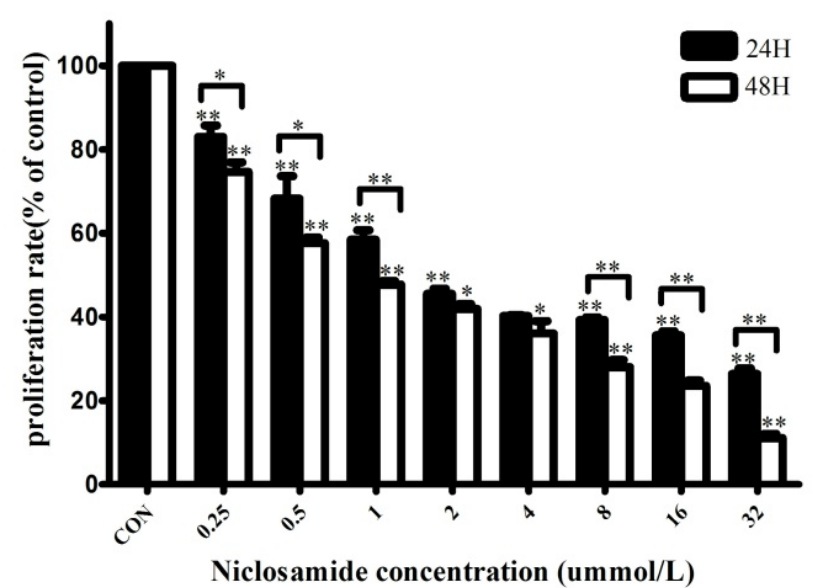

B

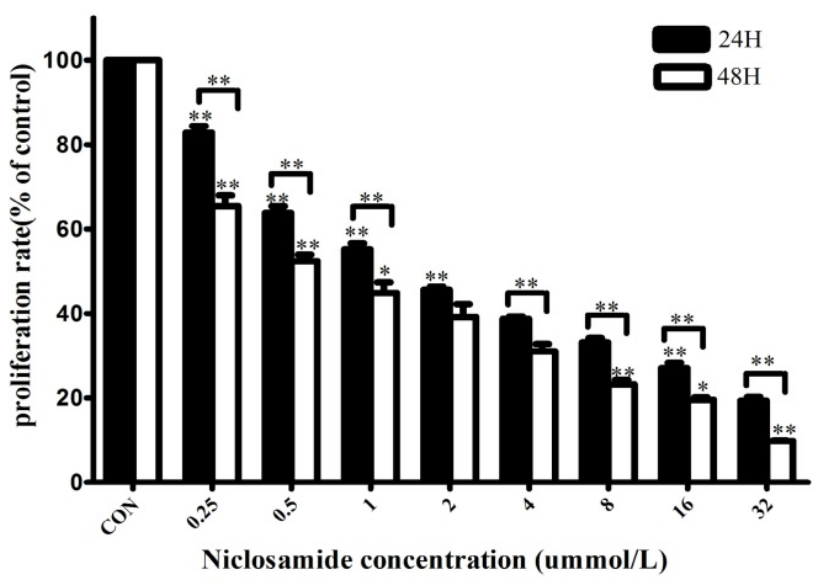

Figure 1. Effects of niclosamide on the proliferation of CNE-2 (A) and HONE-1 (B) cells. NPC cells were treated with niclosamide at the indicated concentrations $(0,0.25,0.5,1,2,4,8,16,32 \mathrm{umol} / \mathrm{L})$ for 24 or $48 \mathrm{~h}$, and the proliferation rates were then determined by CCK-8 assay. Niclosamide significantly reduced the proliferation of these cell lines in a dose- and time-dependent manner. All data are presented as the mean values \pm SD from three independent experiments. Cell proliferation in the untreated control cells was assigned as $100 \%$. $* \mathrm{P}<0.05 ; * * \mathrm{P}<0.01$ vs. control; bars, SD. 

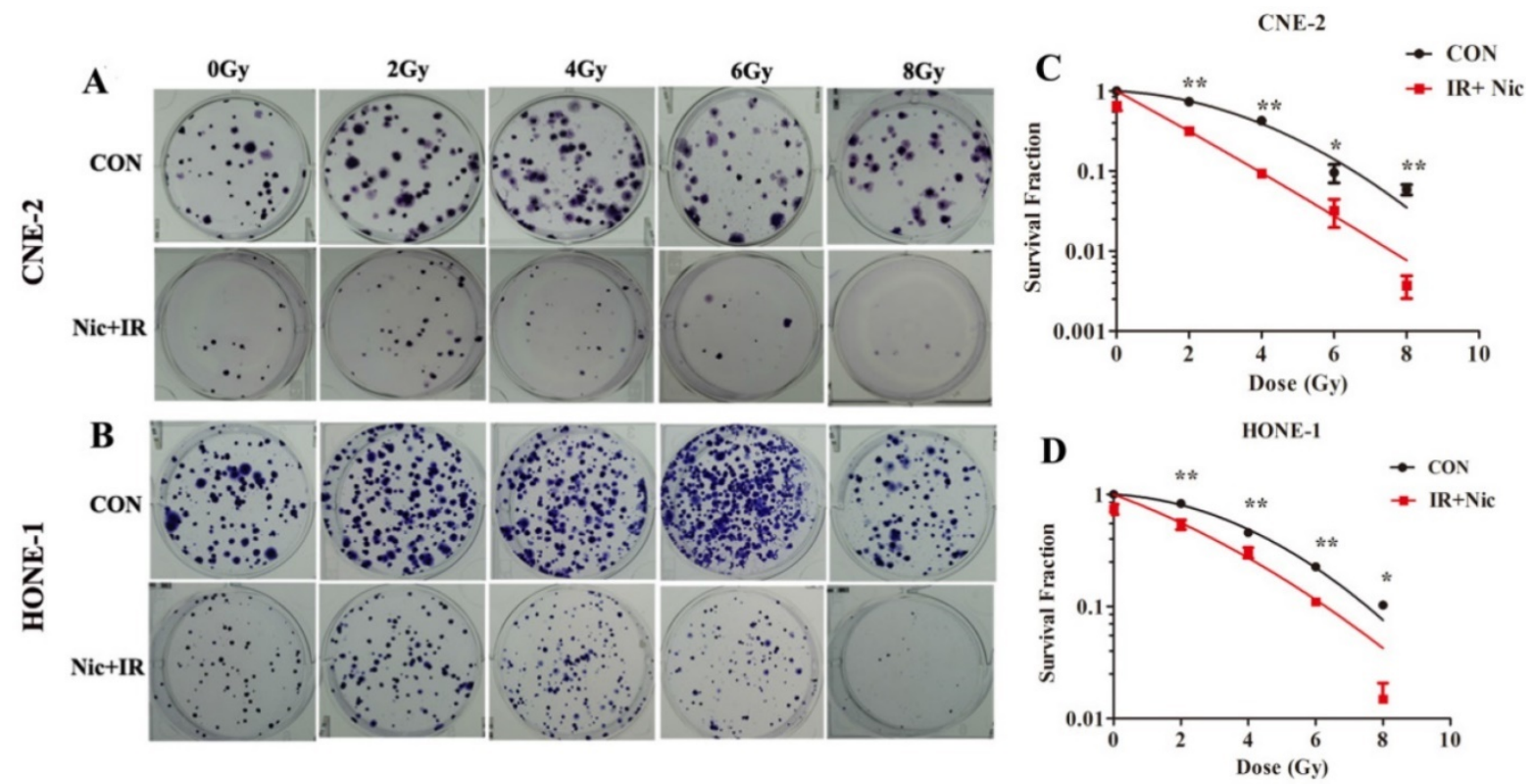

Figure 2. Effects of niclosamide pretreatment followed by irradiation on the colony formation ability of NPC cells. Cells were treated with niclosamide or irradiation alone or pretreated with niclosamide for $24 \mathrm{~h}$ followed by 2 to $8 \mathrm{~Gy}$ irradiation and allowed to grow for 10 days. Representative images of colony formation of CNE-2 (A) and HONE-1 (B) cells are shown. Survival curves of CNE-2 (C) and HONE-1 (D) cells were obtained from the results of the colony forming assays. Survival curves were fitted using the multi-target click model. Niclosamide markedly enhanced the radiosensitivity of these NPC cell lines with a SER of 2.33 for CNE-2 and 1.54 for HONE-1, respectively. All data are presented as mean values \pm SD from three independent experiments. ${ }^{*} \mathrm{P}<0.05$, ${ }^{* *} \mathrm{P}<0.01$. IR, irradiation; Nic, niclosamide; IR + Nic, irradiation plus niclosamide.

\section{Niclosamide reduced the colony forming ability of NPC cells after exposure to irradiation}

To further determine whether niclosamide could increase the radiosensitivity of NPC cells, colony formation assay, which is a gold standard assessment of radiosensitivity, was performed. The radiationsurvival curves of these two cell lines were presented in Fig. $2 \mathrm{~A}$ and $2 \mathrm{~B}$, in which cells were exposed to radiation at $0,2,4,6$ or $8 \mathrm{~Gy}$ with or without 0.8 $\mu \mathrm{mol} / \mathrm{L}$ niclosamide pretreatment, and the surviving colonies were counted after 10 days' incubation [18]. The radiation sensitivity was expressed as the surviving fraction at a clinically relevant dose of 2 Gy (SF2) and sensitization enhancement ratios (SER) were calculated. The SF2 for CNE-2 cells following IR and IR plus $0.8 \mathrm{umol} / \mathrm{L}$ niclosamide were 0.734 and 0.315 respectively (Fig. 2C), and the SF2 for HONE-1 were 0.829 and 0.538 , respectively (Fig. 2D). Consistently, niclosamide markedly enhanced the radiosensitivity of cell lines with SERs of 2.33 for CNE-2 and 1.54 for HONE-1 cells, respectively. Consequently, our data indicates that niclosamide can significantly enhance the radiosensitivity of NPC cells.

\section{Niclosamide enhanced radiation-induced apoptosis of NPC cells}

In order to investigate whether niclosamide induced NPC cells an apoptotic response, CNE-2 and
HONE-1 cells were exposed to niclosamide at the concentration of $0.8 \mu \mathrm{mol} / \mathrm{L}$ and $0.5 \mu \mathrm{mol} / \mathrm{L}$, respectively, for $24 \mathrm{~h}$ and then in the presence or absence of irradiation, flow cytometry was used to assess apoptotic cells $36 \mathrm{~h}$ later. Our results demonstrated that apoptosis was induced in the CNE-2 and HONE-1 cells treated with niclosamide alone (Fig. 3). The results also showed that treatment with niclosamide and radiation significantly increased cell apoptosis compared with cells exposed to radiation or niclosamide alone, with a $\mathrm{p}$ value $<0.0001$ and $<0.0001$ in CNE-2 and HONE-1 cells, respectively. The apoptotic rate of the control, niclosamide, radiation and niclosamide plus radiation were $6.75 \pm 0.212 \%, 25.35 \pm 2.19 \%$, $10.65 \pm 2.47 \%$, $55.2 \pm 9.79 \%$ in CNE-2 cells and $13.53 \pm 0.55 \%$, $37.86 \pm 1.66 \%, 28.8 \pm 2.17 \%, 66.3 \pm 3.82 \%$ in HONE-1 cells, respectively (Fig. 3). Thus, our data demonstrated that the combined treatment of niclosamide with radiation significantly enhanced the apoptosis in both CNE-2 and HONE-1 cells.

\section{Niclosamide increased $\mathrm{Y}-\mathrm{H} 2 \mathrm{AX}$ foci formation in response to irradiation.}

Gamma-H2AX is a well-documented marker of DSBs $^{15}$. Immunocytochemical analysis using anti- $\gamma-\mathrm{H} 2 \mathrm{AX}$ antibodies was conducted in order to determine the effects of niclosamide on DNA damage. As shown in representative micrographs, the number of $\mathrm{\gamma}-\mathrm{H} 2 \mathrm{AX}$ foci was clearly distinguished after the four treatments (Fig $4 \mathrm{~A} \& \mathrm{~B}$ ). The mean number of 
$\mathrm{Y}-\mathrm{H} 2 \mathrm{AX}$ foci was shown to be significantly increased in the cells treated with irradiation alone, while $\mathrm{Y}-\mathrm{H} 2 \mathrm{AX}$ foci in the cells exposed to niclosamide $(0.8$ $\mu \mathrm{mol} / \mathrm{L}, 24 \mathrm{~h}$ ) prior to irradiation were dramatically increased over a $1 \mathrm{~h}, 4 \mathrm{~h}$ and $12 \mathrm{~h}$ time course in both
CNE-2 (Fig 4C) and HONE-1 cells (Fig 4D). Consequently, the results implied that niclosamide combined with irradiation significantly increased the DSBs compared to the cells treated with niclosamide or irradiation alone.
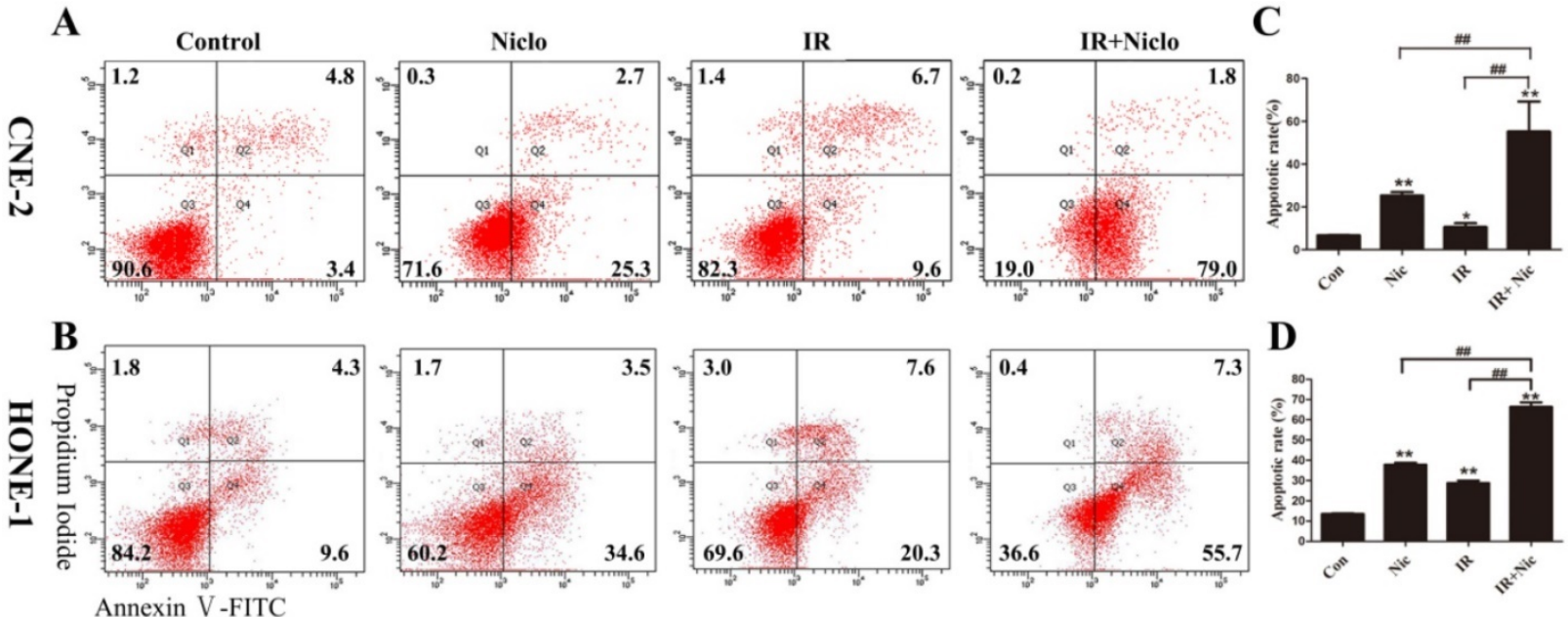

Figure 3. Flow cytometric analysis of niclosamide-induced apoptosis in CNE-2 (A and C) and HONE-1 (B and D) cell using Annexin-V-FITC/PI staining. Cells were incubated with niclosamide at a concentration of $0.8 \mu \mathrm{mol} / \mathrm{L}(\mathrm{CNE}-2)$ or $0.5 \mu \mathrm{mol} / \mathrm{L}$ (HONE-1) for $24 \mathrm{~h}$ and then replaced with refresh medium for $36 \mathrm{~h}$. Early apoptotic cells and late apoptosis cells were determined by Annexin $\mathrm{V}(+) / \mathrm{PI}(-)$ staining $(\mathrm{Q} 4)$ in the lower right quadrant and Annexin $\mathrm{V}(+) / \mathrm{PI}(+)$ staining $(\mathrm{Q} 2)$ in the upper right quadrant. Data are representative of one of three similar experiments. * representated $\mathrm{P}$ value of treatment groups vs control group, \# was IR vs IR+Nic. *P<0.05, $* * P<0.01$; $\# \mathrm{P}<0.05, \# \mathrm{P}<0.01$. IR, irradiation; IR+Nic, irradiation plus niclosamide.
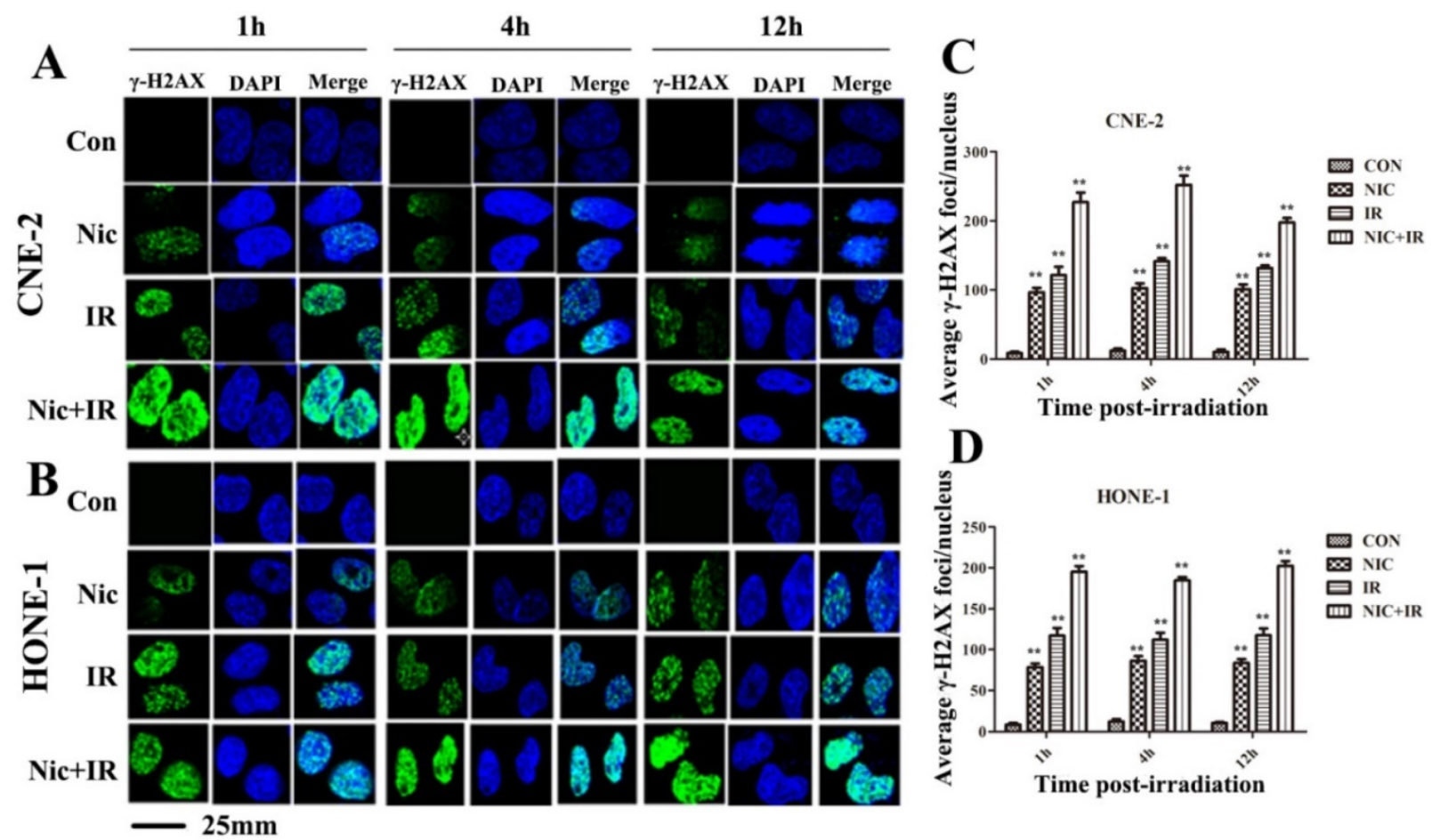

Figure 4. Effects of niclosamide pretreatment on irradiation-induced $\mathbf{Y}-\mathrm{H} 2 \mathrm{AX}$ foci formation in NPC cells. Cells growing on coverslips in 6-well plates were treated with niclosamide or irradiation alone or pretreated with niclosamide for $24 \mathrm{~h}$ followed by 6 -Gy irradiation and then stained for $\mathrm{Y}$ - $\mathrm{H} 2 \mathrm{AX}$ foci. Representative images of $\mathrm{Y}-\mathrm{H} 2 \mathrm{AX}$ foci in CNE-2 (A) and HONE-1 (B) cells were shown. Quantification of the average numbers of $\mathrm{Y}-\mathrm{H} 2 \mathrm{AX}$ foci per nucleus at the indicated time point after various treatments (C and D). The mean number of $\mathrm{Y}-\mathrm{H} 2 \mathrm{AX}$ foci per cells treated with a combination of niclosamide and irradiation was significantly increased compared with irradiation or niclosamide alone in the different treatments followed by a 1- to 12-h post-irradiation duration. The average number of $\mathrm{Y}$-H2AX foci per nucleus was determined randomly scoring 50 nuclei per sample, and each experiment was repeated at least three times. Merged foci were quantified and plotted for CNE-2 (C) and HONE-1 (D) cells at 1, 4 and $12 \mathrm{~h}$ post-irradiation. Columns: mean values $\pm \mathrm{SD}$ of three independent experiments. $* \mathrm{P}<0.05, * * \mathrm{P}<0.01$. vs. other groups at the same point of time. 


\section{Niclosamide affected the expression of DNA damage repair-associated proteins in response to irradiation}

We next explored the impact of niclosamide on the expression of DNA damage repair-related proteins by western blot analysis. The protein levels of DNA damage repair-associated proteins in CNE-2

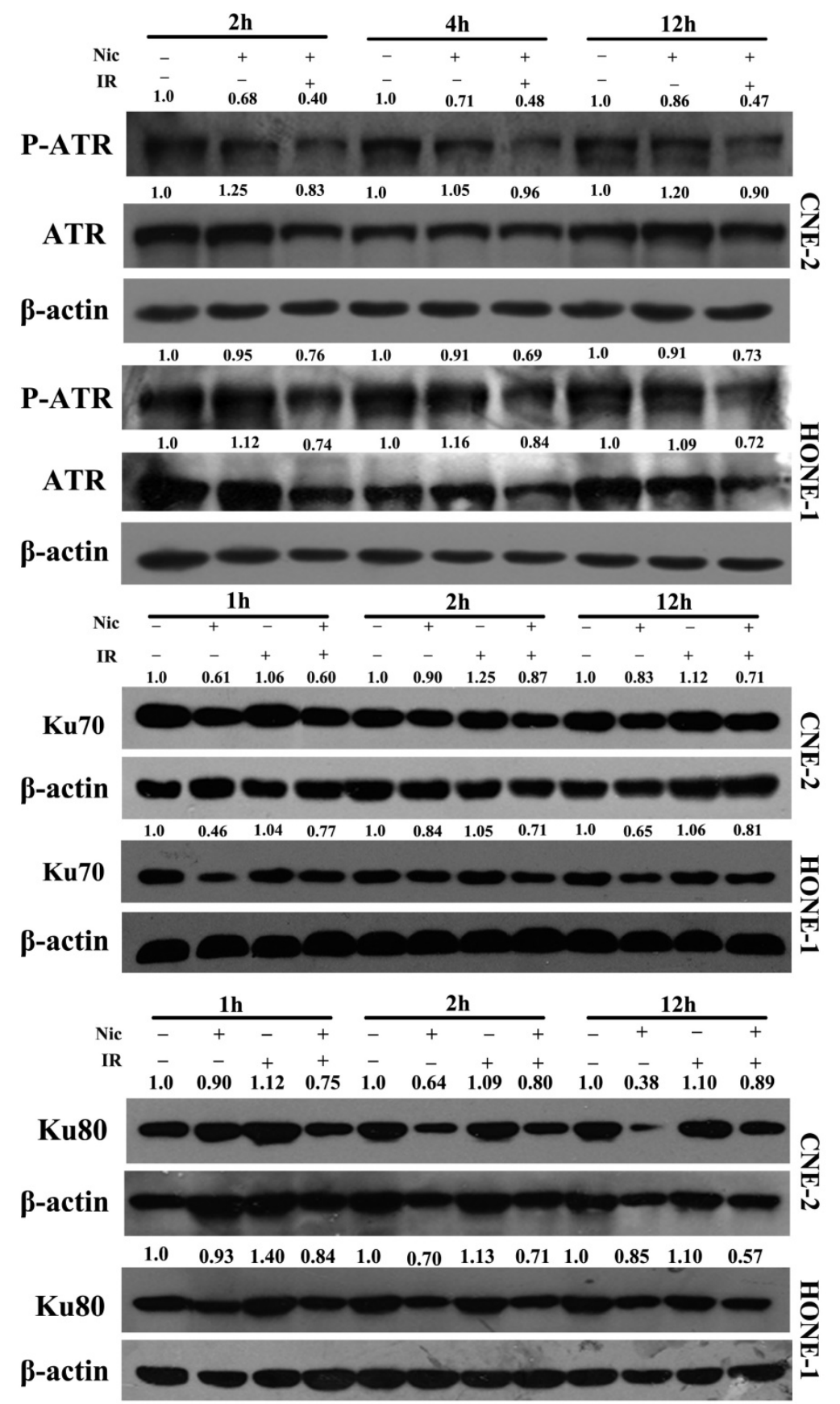

Figure 5. Effects of niclosamide combined with irradiation on the molecules associated with the NHEJ pathway in NPC cells. Whole cell lysates were prepared and western blot analysis was performed using anti -ATR, -p-ATR, anti-Ku70, or anti-Ku80 for the NHEJ pathway. In both of the cell lines, the expression levels of P-ATR (Ser428) were up-regulated, while the expression levels of ATR were down-regulated by niclosamide. The expression levels of Ku70 and Ku80 proteins in niclosamide plus irradiation group were far more effective than control or irradiation alone. The representative results are shown. The ratios above each blot are expressed as the intensity of the blot relative to that of the untreated control. and HONE-1 cells under various conditions are shown in Fig. 5. Clearly, in both cell lines, the expression levels of p-ATR (Ser428) was up-regulated, and that ATR was down-regulated by irradiation, whereas the expression levels of p-ATR(Ser428) and ATR were down-regulated by niclosamide (Fig 5). Furthermore, the expression level of Ku70/80 protein was also significantly down-regulated over time after niclosamide treatment in both CNE-2 and HONE-1 cells by the combination of niclosamide with irradiation or niclosamide alone (Fig 5).

\section{Niclosamide inhibited the transcription but not the translocation of $\mathrm{Ku} \mathbf{7 0 / 8 0}$ proteins}

To test whether niclosamide regulates $\mathrm{Ku} 70 / 80$ expression at the transcription level, we detected Ku70/80 mRNA by quantitative real-time PCR analyses in CNE-2 and HONE-1 cells treated with niclosamide at a concentration of $0.8 \mathrm{umol} / \mathrm{L}$ for $24 \mathrm{~h}$. As shown in (Fig. 6), niclosamide significantly reduced the expression of Ku70/80 mRNA in both CNE-2 (Fig 6B) and HONE-1 (Fig 6D) cells. Consistently, the expression of $\mathrm{Ku} 70 / 80$ protein was also significantly down-regulated in these cells treated with niclosamide at a concentration of $0.8 \mathrm{umol} / \mathrm{L}$ for $24 \mathrm{~h}$ (Fig.6A\&C). We further analyzed whether niclosamide influenced the localization of Ku70/80 proteins in CNE-2 and HONE-1 cells. As shown in representative micrographs (Fig 7), the number of $\mathrm{Ku} 70 / 80$ foci was clearly distinguished after the different treatments. Ku70/80 subunits were mainly localized to the nuclei before irradiation in both cell lines. The intensity of Ku70/80 nuclear staining was increasing from 1 to $6 \mathrm{~h}$ after irradiation. However, the mean number of $\mathrm{Ku} 70 / 80$ foci per cell was declined in the cells treated with niclosamide $(0.8 \mathrm{uM})$ alone and irradiation alone could significantly increase the Ku70/80 foci intensity compared to control group, however, niclosamide combined with irradiation significantly reduced the Ku70/80 foci intensity of both CNE-2 and HONE-1 cells. Therefore, these data indicated that niclosamide might reduce the $\mathrm{Ku} / 80$ protein by, at least in part, by inhibiting the transcriptional level, but not 

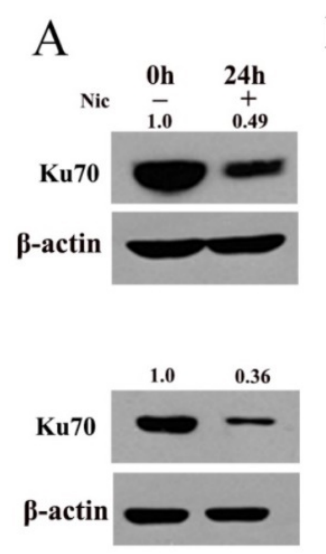
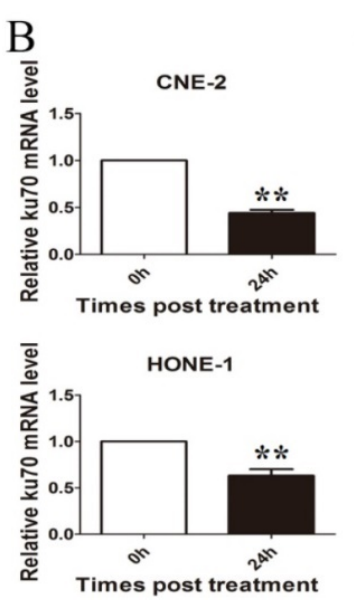

$\mathrm{C}$
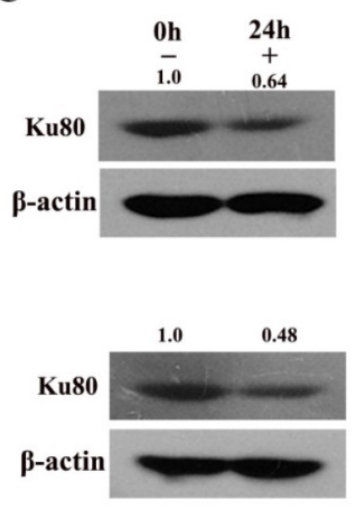

$\mathrm{D}$
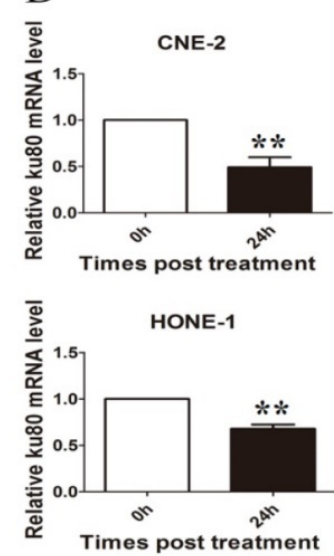

Figure 6. Niclosamide remarkably reduced the expression level of Ku70 and Ku80 mRNA and protein as detected by qRT-PCR or western blotting. (A) Niclosamide significantly reduced the protein level (A) and mRNA (B) level of Ku70 in CNE-2 and HONE-1 cells. Niclosamide significantly reduced the protein level (C) and mRNA (D) level of Ku80 in CNE-2 and HONE-1 cells. The ratios above each blot are expressed as the intensity of the blot relative to that of the untreated control. $* * P<0.01$ vs. control; bars, SD.

the nuclear and cytoplasm location.

\section{Discussion}

The present study showed that niclosamide could markedly suppress the proliferation of NPC cells (Fig. 1). Furthermore, colony formation assay demonstrated that niclosamide combined with irradiation significantly decreased the clonogenic formation abilities and enhanced the radiosensitivity of NPC cells, with a sensitizing enhancement ratio (SER) of 2.33 and 1.54 in CNE-2 and HONE-1 cells, respectively (Fig. 2). Niclosamide in combination with irradiation significantly increased the anti-tumor effect of irradiation by inducing the apoptosis of NPC cells (Fig. 3). Consistently, Lee et al $^{16}$ demonstrated that niclosamide radiosensitized human lung cancer cells through activation of P38MAPK-c-Jun axis. You et $\mathrm{al}^{17}$ demonstrated that inhibition of the STAT3/ $\mathrm{Bcl} 2 / \mathrm{Bcl}-\mathrm{XL}$ pathway by niclosamide effectively overcame acquired radioresistance in vitro and in vivo. In addition, niclosamide was found to sensitize triplenegative breast cancer cells to irradiation, prevent Wnt3a-induced radioresistance and overcome $\beta$ catenin-induced radioresistance in triple-negative breast cancer cells ${ }^{18}$. To sum up, our observation found for the first time that niclosamide could sensitize the nasopharyngeal carcinoma cells to irradiation.

Ionizing radiation has a powerful killing effect to the nasopharyngeal carcinoma ${ }^{19}$. This anticancer effect of ionizing radiation causes double strands breaks (DSBs) in DNA which are the most lethal type of DNA damage. If cancer cells recover from such damage, they will survive. However, if the DNA damage remains unrepaired or is incorrectly repaired prior to DNA replication or mitosis, DSBs can result in apoptosis, mitotic cell death and permanent cell cycle significantly increased over time $(1,4,12 \mathrm{~h})$ after irradiation. Notably, although irradiation or niclosamide alone could induce nuclear $\gamma$-H2AX foci formation, niclosamide plus irradiation markedly induced an increased number of nuclear $\gamma-\mathrm{H} 2 \mathrm{AX}$ foci compared to either irradiation or niclosamide treatment (Fig. 4). Therefore, niclosamide plus irradiation remarkably increased the DNA damage in NPC cells, suggesting that niclosamide might sensitize NPC cells to ionizing radiation through aggravation of DNA damage.

Several pathways have been involved in the repair of DSBs, including homologous recombination (HR) and non-homologous end-joining (NHEJ). The classical NHEJ pathway repairs a predominant fraction of DSBs in mammalian cells ${ }^{22}$. In NHEJ, the DSB ends are bound by the abundant Ku70/80 heterodimer, which in turn recruit other NHEJ proteins such as the catalytic subunit of the DNA-dependent protein kinase (DNA-PKcs), ligases and polymerases, and then facilitate the repair process $^{23}$. The roles of $\mathrm{Ku} 70$ and/or Ku80 protein have been widely studied in several malignancies. Veerle $^{24}$ et al comfirmed that partial knockdown of $\mathrm{Ku}$ proteins enhanced the radiosensitivity of human MCF10A cells to X-rays irradiation. Karin ${ }^{25}$ et al found that low protein expression of the Ku70/80 heterodimer predicted good response to radiotherapy in early breast cancer. Tarish et al found that patients receiving neoadjuvant chemical castration treatment before radiotherapy had reduced amounts of Ku70 protein, impaired radiotherapy-induced NHEJ activity, and higher amounts of unrepaired DSBs, and thus improved the therapeutic response in prostate cancer $^{26}$. Leifler et al reported that low expression of $\mathrm{Ku} 70 / 80$ predicted good response to radiotherapy in early breast cancer ${ }^{27}$. Similar results were reported in 
nasopharyngeal carcinoma. Lee et al found that the patients with low Ku70 protein level showed a higher 5-year locoregional control than those with high Ku70 protein level, while a higher proportion of Ku70-positive tumor cells was closely associated with poor response to radiation therapy and high risk of loco-regional recurrence ${ }^{28}$. Given the vital role of $\mathrm{Ku} 70 / 80$ heterodimer in NHEJ and radiotherapy response, we suggested that impaired DSB repair could potentially be the mechanistic explanation for the increased radiosensitivity by nicolosamide.

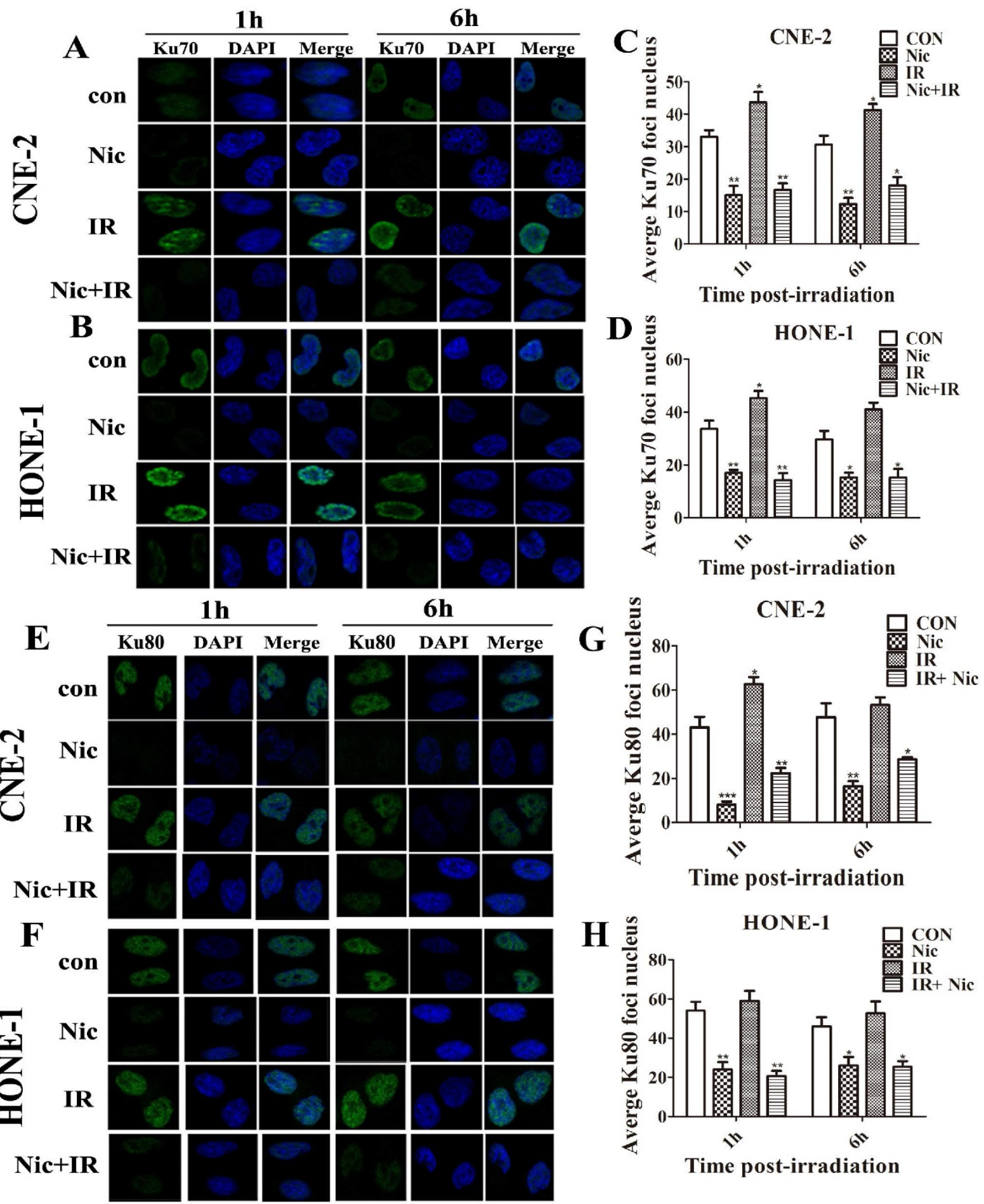

Figure 7. Effects of niclosamide pretreatment on irradiation-induced Ku70 and Ku80 foci formation in NPC cells. Cells growing on coverslips in 6-well plates were treated with niclosamide or irradiation alone or pretreated with niclosamide for $24 \mathrm{~h}$ followed by 6-Gy irradiation and then stained for Ku70 or Ku80 foci. Representative images of Ku70 and Ku80 foci in CNE-2 (A\&E) and HONE-1 (B\&F) cells. Quantification of the average numbers of Ku70 and Ku80 foci per nucleus at the time points as indicated after various treatments. The mean number of $\mathrm{Ku} 70$ and Ku80 foci per cells treated with a combination of niclosamide and irradiation was significantly increased compared with irradiation or control alone in the different treatments followed by a 1- to 6-h post-irradiation duration. The average numbers of Ku70 or Ku80 foci per nucleus was determined randomly scoring 50 nuclei per sample, and each experiment was repeated at least three times. Merged foci were quantified and plotted for CNE-2 (C, G) and HONE-1 (D , H) cells at 1 , and $6 \mathrm{~h}$ post-irradiation. 
To verify this hypothesis, we investigated the effects of niclosamide on the molecules involved in NHEJ pathways by western blot assay. Our results showed that niclosamide plus irradiation or niclosamide alone significantly reduced the expression levels of Ku70 and Ku80 proteins and reduced the level of ATR kinase and the phosphorylated form of ATR (pATR) (Fig. 5). In addition, immunofluorescent staining was used to detect the localization of Ku70/Ku80 in NPC cells. We observed that $\mathrm{Ku} 70 / 80$ proteins were localized in the nuclei of NPC cells and irradiation could slightly increase the intensity of $\mathrm{Ku} 70$ or $\mathrm{Ku} 80$ protein staining. However, niclosamide alone or niclosamide plus irradiation markedly reduced the numbers of nuclear $\mathrm{Ku} 70 / \mathrm{Ku} 80$ foci compared to either irradiation or control group (Fig. 6). Consequently, we surmised that niclosamide might enhance the cytotoxical effects of radiation by reducing $\mathrm{Ku} 70 / \mathrm{Ku} 80$ protein level, which play a critical role in regulating NHEJ in NPC cells.

To further explore how niclosamide reduce the expression of $\mathrm{Ku} 70$ and $\mathrm{Ku} 80$ proteins, quantitative real-time PCR analyses and western blotting were used to examine the expression of Ku70/80 mRNA and proteins after niclosamide treatment. We found that the mRNA levels of Ku70/Ku80 treated with niclosamide were significantly lower than these of control group (Fig. 7A). Consistently, the expression of $\mathrm{Ku} 70 / \mathrm{Ku} 80$ proteins was also reduced by niclosamide compared with the control group (Fig. 7B). These results suggested that niclosamide might impair the NHEJ pathways after radiation via inhibition of Ku70/80 transcription in NPC cells.

In summary, the present study demonstrated that niclosamide could inhibit the growth of nasopharyngeal carcinoma cells and sensitize NPC cells to radiation by inhibition of NHEJ pathway via suppressing the transcription of Ku70/80. These data imply that niclosamide might be a radiation sensitizer candidate for the treatment of NPC.

\section{Acknowledgements}

This work was supported by the funding from National Natural Science Foundation of China (No. 81201736), Natural Science Foundation of Guangdong Province, China (No. 2015A030310460), The Research Fund of Guangdong Medical University (2X14031).

\section{Competing Interests}

The authors have declared that no competing interest exists.

\section{References}

1. Chia WK, Teo M, Wang WW, et al. Adoptive T-cell transfer and chemotherapy in the first-line treatment of metastatic and/or locally recurrent nasopharyngeal carcinoma. MOL THER 2014;22:132-9.

2. Wang W, Feng M, Fan Z, Li J, Lang J. Clinical outcomes and prognostic factors of 695 nasopharyngeal carcinoma patients treated with intensity-modulated radiotherapy. BIOMED RES INT 2014;2014:814948.

3. $\mathrm{Tu} \mathrm{Z}, \mathrm{Xu} \mathrm{B}, \mathrm{Qu} \mathrm{C}$, et al. BRCC3 acts as a prognostic marker in nasopharyngeal carcinoma patients treated with radiotherapy and mediates radiation resistance in vitro. RADIAT ONCOL 2015;10:123

4. Tanowitz HB, Weiss LM, Wittner M. Diagnosis and treatment of intestinal helminths. I. Common intestinal cestodes. Gastroenterologist 1993;1:265-73.

5. Merschjohann K, Steverding D. In vitro trypanocidal activity of the anti-helminthic drug niclosamide. EXP PARASITOL 2008;118:637-40.

6. Li R, You S, Hu Z, et al. Inhibition of STAT3 by niclosamide synergizes with erlotinib against head and neck cancer. PLOS ONE 2013;8:e74670.

7. King ML, Lindberg ME, Stodden GR, et al. WNT7A/beta-catenin signaling induces FGF1 and influences sensitivity to niclosamide in ovarian cancer. ONCOGENE 2015;34:3452-62.

8. Liu C, Lou W, Zhu Y, et al. Niclosamide inhibits androgen receptor variants expression and overcomes enzalutamide resistance in castration-resistant prostate cancer. CLIN CANCER RES 2014;20:3198-210.

9. Karakas D, Cevatemre B, Aztopal N, Ari F, Yilmaz VT, Ulukaya E. Addition of niclosamide to palladium(II) saccharinate complex of terpyridine results in enhanced cytotoxic activity inducing apoptosis on cancer stem cells of breast cancer. Bioorg Med Chem 2015·23.5580-6.

10. Xiang D, Yuan Y, Chen L, Liu X, Belani C, Cheng H. Niclosamide, an anti-helminthic molecule, downregulates the retroviral oncoprotein Tax and pro-survival Bcl-2 proteins in HTLV-1-transformed T lymphocytes. Biochem Biophys Res Commun 2015;464:221-8.

11. Osada T, Chen M, Yang $X Y$, et al. Antihelminth compound niclosamide downregulates Wnt signaling and elicits antitumor responses in tumors with activating APC mutations. CANCER RES 2011;71:4172-82.

12. Fonseca BD, Diering GH, Bidinosti MA, et al. Structure-activity analysis of niclosamide reveals potential role for cytoplasmic $\mathrm{pH}$ in control of mammalian target of rapamycin complex 1 (mTORC1) signaling. J BIOL CHEM 2012:287:17530-45

13. Khanim FL, Merrick BA, Giles HV, et al. Redeployment-based drug screening identifies the anti-helminthic niclosamide as anti-myeloma therapy that also reduces free light chain production. BLOOD CANCER J 2011;1:e39.

14. $\mathrm{Xu} \mathrm{Z,} \mathrm{Fang} \mathrm{S,} \mathrm{Zuo} \mathrm{Y,} \mathrm{et} \mathrm{al.} \mathrm{Combination} \mathrm{of} \mathrm{pigment} \mathrm{epithelium-derived} \mathrm{factor}$ with radiotherapy enhances the antitumor effects on nasopharyngeal carcinoma by downregulating vascular endothelial growth factor expression and angiogenesis. CANCER SCI 2011;102:1789-98.

15. Branzei D, Foiani M. Regulation of DNA repair throughout the cell cycle. Nat Rev Mol Cell Biol 2008;9:297-308.

16. Lee S, Lim MJ, Kim MH, et al. An effective strategy for increasing the radiosensitivity of Human lung Cancer cells by blocking Nrf2-dependent antioxidant responses. Free Radic Biol Med 2012;53:807-16.

17. You S, Li R, Park D, et al. Disruption of STAT3 by niclosamide reverses radioresistance of human lung cancer. MOL CANCER THER 2014;13:606-16.

18. Yin L, Gao Y, Zhang X, et al. Niclosamide sensitizes triple-negative breast cancer cells to ionizing radiation in association with the inhibition of Wnt/beta-catenin signaling. ONCOTARGET 2016;7:42126-38.

19. Pan F, Ruan Z, Li J, et al. Radiotherapy combined docetaxel and oxaliplatin chemotherapy is effective in patients with locally advanced nasopharyngeal carcinoma. MED ONCOL 2015·32.252

20. Qi D, Hu Y, Zhang Y, Peng T, Ji W. Effect of Ku70 expression on radiosensitivity in renal carcinoma 786-O cells. CANCER CELL INT 2014;14:44.

21. Sak A, Stuschke M. Use of gammaH2AX and other biomarkers of double-strand breaks during radiotherapy. SEMIN RADIAT ONCOL 2010:20:223-31.

22. Mahaney BL, Meek K, Lees-Miller SP. Repair of ionizing radiation-induced DNA double-strand breaks by non-homologous end-joining. BIOCHEM J 2009;417:639-50.

23. Olsen BB, Wang SY, Svenstrup TH, Chen BP, Guerra B. Protein kinase CK2 localizes to sites of DNA double-strand break regulating the cellular response to DNA damage. BMC MOL BIOL 2012;13:7.

24. Vandersickel V, Mancini M, Slabbert J, et al. The radiosensitizing effect of Ku70/80 knockdown in MCF10A cells irradiated with X-rays and $\mathrm{p}(66)+\mathrm{Be}(40)$ neutrons. RADIAT ONCOL 2010;5:30

25. Soderlund LK, Queseth S, Fornander T, Askmalm MS. Low expression of $\mathrm{Ku} 70 / 80$, but high expression of DNA-PKCs, predict good response to radiotherapy in early breast cancer. INT J ONCOL 2010;37:1547-54

26. Tarish FL, Schultz N, Tanoglidi A, et al. Castration radiosensitizes prostate cancer tissue by impairing DNA double-strand break repair. SCI TRANSL MED 2015:7:311r-312r.

27. Soderlund LK, Queseth S, Fornander T, Askmalm MS. Low expression of Ku70/80, but high expression of DNA-PKcs, predict good response to radiotherapy in early breast cancer. INT J ONCOL 2010;37:1547-54.

28. Lee SW, Cho KJ, Park JH, et al. Expressions of Ku70 and DNA-PKcs as prognostic indicators of local control in nasopharyngeal carcinoma. Int J Radiat Oncol Biol Phys 2005;62:1451-7. 